\title{
miR-21, miR-221 and miR-222 expression and prostate cancer recurrence among obese and non-obese cases
}

\begin{abstract}
Ernest K Amankwah ${ }^{1}$, Evelyn Anegbe ${ }^{1}$, Hyun Park ${ }^{1}$, Julio Pow-Sang ${ }^{2}$, Ardeshir Hakam ${ }^{3}$ and Jong Y Park ${ }^{1}$
Recent evidence shows that certain microRNAs (miRNAs) play a role in both obesity and prostate cancer recurrence, but the association between the expression of these miRNAs and obesity in prostate cancer recurrence is unknown. In this study, we examined the effect of the interaction between obesity and miR-21, miR-221 or miR-222 expression on prostate cancer recurrence among 28 recurrent and 37 non-recurrent prostate cancer cases. miRNA expression was determined using quantitative real-time polymerase chain reaction. Cox proportional hazard models adjusting for age at diagnosis, clinical stage and Gleason score were used to estimate hazard ratios (HR) and $95 \%$ confidence intervals $(95 \% \mathrm{Cl})$ for recurrence free survival. A significantly $(P=0.014)$ higher proportion of recurrent cases (78.6\%) than non-recurrent cases (48.6\%) had a low expression of miR-21 and the difference was more prominent in obese than non-obese patients. Multivariate analysis showed that the expression of miR-21 was an independent risk factor for recurrence in obese $(\mathrm{HR}=6.15,95 \% \mathrm{Cl}=1.04-36.48, P=0.045)$, but not in non-obese $(\mathrm{HR}=1.28,95 \% \mathrm{Cl}=0.30-5.49, P=0.74)$ cases. A significant association with recurrence was not observed for the expression of miR-221 and miR-222. In summary, our findings show that miR-21 is associated with prostate cancer recurrence after radical prostatectomy and suggest that the differential expression of miR-21 is more prominent in obese than in non-obese cases. Future larger studies are warranted to confirm these initial findings and to elucidate the mechanisms involved.
\end{abstract}

Asian Journal of Andrology (2013) 15, 226-230; doi:10.1038/aja.2012.160; published online 28 January 2013

Keywords: miR-21; miR-221; miR-222; miRNA; obesity; prostate carcinoma; recurrence

\section{INTRODUCTION}

Prostate cancer is the most common non-skin malignancy among men in the United States. An estimated 241740 new cases and 28170 deaths related to prostate cancer are expected in $2012 .{ }^{1}$ The majority $(>80 \%)$ of prostate cancer patients are diagnosed at an early stage when the disease is confined to the prostate. ${ }^{2}$ Radical prostatectomy (RP) is the treatment of reference for organ-confined prostate tumors. Unfortunately, about $30 \%$ of patients who undergo RP develop tumor recurrence within 10 years post-surgery. ${ }^{3}$ Currently, the level of prostate-specific antigen, clinical stage and the grade of tumor (Gleason score) are used to estimate prognosis and inform treatment modalities. Although these features are extremely useful in predicting recurrence, they do not account for the varied interindividual outcomes associated with RP. Therefore, biomarkers are needed to supplement clinical and pathological observations to identify patients at greatest risk of developing recurrence after RP.

Expression profiling studies have shown the critical role of microRNAs (miRNAs) in prostate cancer. ${ }^{4}$ However, little is known about miRNA expression and prostate cancer recurrence. To date, only a few studies have investigated miRNA expression and prostate cancer recurrence after radical prostatectomy ${ }^{5-10}$ and these studies suggest that miRNAs, including miR-21, miR-221 and miR-222, are potential prognostic markers for recurrence. These same three miRNAs have also been implicated in obesity. ${ }^{11}$ Since obesity is an important predictor for prostate cancer recurrence, ${ }^{12}$ it may modify the association between miRNA expression and prostate cancer recurrence. To date, no study has examined the modifying effect of obesity on the association between miRNA expression and prostate cancer recurrence.

In this study, we evaluated the associations between prostate cancer recurrence and the expression of miR-21, miR-221 and miR-222 in tumor samples from 37 non-recurrent and 28 recurrent prostate cancer patients that were treated with radical prostatectomy at the Moffitt Cancer Center. We then examined whether these associations were modified by obesity.

\section{MATERIALS AND METHODS}

Study population

The study included oversampled 28 recurrent and 37 non-recurrent prostate cancer cases, which were histologically confirmed. Participants were treated with radical prostatectomy at the Moffitt Cancer Center (Tampa, FL, USA) between 1987 and 2005 and followed up till 2011. Patients' demographic and clinical information, such as age at diagnosis, body mass index (BMI), clinical stage, Gleason grade, follow-up and recurrence status were obtained from the Moffitt Cancer Registry and medical chart reviews. We investigated two outcomes in this study: recurrence and aggressiveness. A recurrent case was defined by either an elevated prostate-specific

${ }_{1}^{1}$ Department of Cancer Epidemiology, H. Lee Moffitt Cancer Center, Tampa, FL 33612, USA; ${ }^{2}$ Department of GU Oncology, H. Lee Moffitt Cancer Center, Tampa, FL 33612, USA and ${ }^{3}$ Department of Anatomic Pathology, H. Lee Moffitt Cancer Center, Tampa, FL 33612, USA

Correspondence: Dr JY Park (jong.park@moffitt.org)

Received: 12 October 2012; Revised: 19 November 2012; Accepted: 12 December 2012; Published online: 28 January 2013 
antigen level $\left(\geqslant 0.2 \mathrm{ng} \mathrm{ml}^{-1}\right.$ ) after surgical treatment, clinical metastasis or disease specific death. Patients with Gleason scores $\geqslant 7$ or stage $\geqslant$ III were considered to have aggressive prostate cancer. All other patients were categorized as non-aggressive. BMI was categorized as $\leqslant 30 \mathrm{~kg} \mathrm{~m}^{-2}$ (non-obese) and $>30 \mathrm{~kg} \mathrm{~m}^{-2}$ (obese). The study protocol was approved by the Institutional Review Board of the University of South Florida (Tampa, FL, USA).

\section{Tumor samples and RNA extraction}

Total RNA was extracted from formalin-fixed paraffin embedded tissue blocks obtained from the Tissue Core Facility at the Moffitt Cancer Center. The study pathologist (Ardeshir Hakam) screened haematoxylin and eosin stained slides of tissue blocks, selected blocks with high prostate tumor content and marked tumor areas on haematoxylin and eosin slides. RNA was then extracted from macrodissected $10 \mu \mathrm{m}$ thick unstained tumor tissue sections (with $>80 \%$ tumor content) of the same formalin-fixed paraffin embedded block, based on marked haematoxylin and eosin slides. miRNeasy formalin-fixed paraffin embedded total RNA isolation kit (Qiagen, CA, USA) was used for RNA extraction. Extraction was performed according to the manufacturer's recommendations and total RNA was quantified using nanodrop spectrophotometer.

\section{Quantitative real-time polymerase chain reaction (PCR)}

miRNA expression was quantified in tissue samples with TaqMan microRNA reverse transcription and assay kits (Applied Biosystems, CA, USA) on an Applied Biosystems 7900HT real-time PCR following the manufacturer's instructions. Briefly, synthesis of complimentary DNA was performed using $10 \mathrm{ng}$ RNA in a total of $15 \mu \mathrm{l}$ reaction. For the quantitative PCR, $0.665 \mu$ l reverse transcribed reaction was used in $10 \mu \mathrm{l}$ reactions and quantitative PCR was performed in MircoAmp fast optical 384-well reaction plate with barcode (Applied Biosystems). miRNA-specific reactions, as well as reactions for U6 small nuclear 2 RNA (RNU6B), which was an internal control, ${ }^{13,14}$ were run for all the samples. Fluorescence from the binding of miRNA-specific and internal control probes to amplification products was measured during the PCR. SDS software (v 2.3; Applied Biosystems) was used to identify cycle threshold $(C t)$ values and $C t$ values $\geqslant 35$ were considered as undetermined.

\section{Statistical analysis}

The expression $\Delta C t$ value for each sample was calculated by normalizing the $C t$ value for each miRNA with RNU6B $(\Delta C t=$ $\left.\Delta C t_{\text {miRNA }}-\Delta C t_{\mathrm{RNU}}\right) . \Delta C t$ values were dichotomized as low/high expression using the median value in the non-recurrent cases as the cutoff point. ${ }^{15}$ The proportions of recurrent and non-recurrent cases with low or high expression of each miRNA were compared using chisquared test or the Fisher's exact test when some of the cells had expected counts of less than five. Similar analyses stratified on BMI ( $\leqslant 30 \mathrm{~kg} \mathrm{~m}^{-2}$ (non-obese) and $>30 \mathrm{~kg} \mathrm{~m}^{-2}$ (obese)) were performed. To determine whether miRNA expression was associated with time to recurrence, we used Kaplan-Meier curves based on the median miRNA expression and a comparison between the curves was determined using the log rank test. Cox proportional hazard models adjusting for age at diagnosis (continuous), stage ( 1 and $2 v s 3$ and 4 ) and Gleason score $(<7 v s \geqslant 7)$ were used to estimate hazard ratios (HR) and $95 \%$ confidence intervals $(95 \% \mathrm{CI})$ for recurrence free survival. Recurrence free survival was defined as the time from surgery date to first documentation of prostate cancer recurrence or prostate cancer specific-death. Logistic regression models were used to estimate odds
Table 1 Demographics and clinical characteristics of study participants

\begin{tabular}{lcc}
\hline & $\begin{array}{c}\text { No recurrence } \\
(\mathrm{n}=37)\end{array}$ & $\begin{array}{c}\text { Recurrence } \\
(\mathrm{n}=28)\end{array}$ \\
\hline $\begin{array}{l}\text { Median age at diagnosis (range), year } \\
\mathrm{BMI}, n(\%)\end{array}$ & $59(47-75)$ & $57(46-75)$ \\
$\quad 330 \mathrm{~kg} \mathrm{~m}^{-2}$ & $29(78.4)$ & $16(57.1)$ \\
$>30 \mathrm{~kg} \mathrm{~m}^{-2}$ & $8(21.6)$ & $12(42.9)$ \\
Stage, $n(\%)$ & & \\
$\quad 1$ and 2 & $25(67.6)$ & $17(60.7)$ \\
3 and 4 & $7(18.9)$ & $10(35.7)$ \\
$\quad$ Missing & $5(13.5)$ & $1(3.6)$ \\
Gleason, $n(\%)$ & & \\
$\quad<7$ & $20(54.1)$ & $8(28.6)$ \\
$\quad \geqslant 7$ & $13(35.1)$ & $15(53.6)$ \\
$\quad$ Missing & $4(10.8)$ & $5(17.8)$ \\
Median time to recurrence (month) & - & $50.9(2.7-114.5)$ \\
Median follow-up duration (month) & $95.1(9.1-254.3)$ & - \\
\hline
\end{tabular}

Abbreviation: BMI, body mass index.

ratios (OR) and 95\% CI for the association between miRNA expression and aggressive prostate cancer. All statistical analyses were performed using SAS v 9.2 (SAS institute, Cary, NC, USA) and $P<0.05$ was considered statistically significant.

\section{RESULTS}

The characteristics of the study participants are summarized in Table 1. The median age of non-recurrent (59 years) and recurrent (57 years) patients were similar. As expected, a higher proportion of recurrent than non-recurrent cases was obese $\left(\mathrm{BMI}>30 \mathrm{~kg} \mathrm{~m}^{-2}\right)$, or diagnosed with late stage (stages 3 and 4) and higher grade (Gleason score $\geqslant 7$ ) tumors. Among recurrent cases the median time to recurrence was 50.9 months and for the non-recurrent cases the median follow-up time was 95.1 months.

Table 2 shows the proportion of non-recurrent and recurrent cases that had low or high expression of miRNAs. The proportion of recurrent cases $(78.6 \%)$ that showed low expression of miR-21 was significantly higher than that of non-recurrent cases $(48.6 \%)(P=0.014)$. Expression analyses stratified by BMI (non-obese $\leqslant 30 \mathrm{~kg} \mathrm{~m}^{-2}$ and obese $>30 \mathrm{~kg} \mathrm{~m}^{-2}$ ) revealed that the differential expression observed for miR-21 was more prominent in obese than in non-obese cases. Among obese patients, the proportion of recurrent cases $(83.3 \%)$ with low expression of miR-21 was significantly $(P=0.019)$ higher than that of non-recurrent cases $(25.0 \%)$. A significant difference was not observed in the non-obese group for miR-21 or for the expression of miR-221 and miR-222 for all subjects or stratified by BMI (Table 2).

Kaplan-Meier survival analysis showed a significant increased recurrence $(\log$-rank $P<0.0001)$ in patients with decreased expression of miR-21 (Figure 1), but not for miR-221 ( $\log$-rank $P=0.57$ ) or miR-222 (log-rank $P=0.24$ ). Age-adjusted Cox regression analysis revealed that cases with a lower expression (compared to those with a higher expression) of miR-21 had an increased risk of recurrence $(\mathrm{HR}=3.31,95 \% \mathrm{CI}=1.32-8.34, P=0.011)$ that was more prominent in obese $(\mathrm{HR}=5.40,95 \% \mathrm{CI}=1.16-25.10, P=0.031)$ than non-obese $(\mathrm{HR}=2.26,95 \% \mathrm{CI}=0.69-734, P=0.18)$ cases (Table 3). Additional adjustment for stage and Gleason score showed that the expression of miR-21 was an independent risk factor for recurrence in only obese patients $(\mathrm{HR}=6.15,95 \% \mathrm{CI}=1.04-36.48, P=0.045)$. No statistically significant association with recurrence was observed for the expression of miR-221 and miR-222 in the multivariate analysis (Table 3 ). 
Table 2 miR-21, miR-221 and miR-222 expression, prostate cancer recurrence and aggressiveness by body mass index

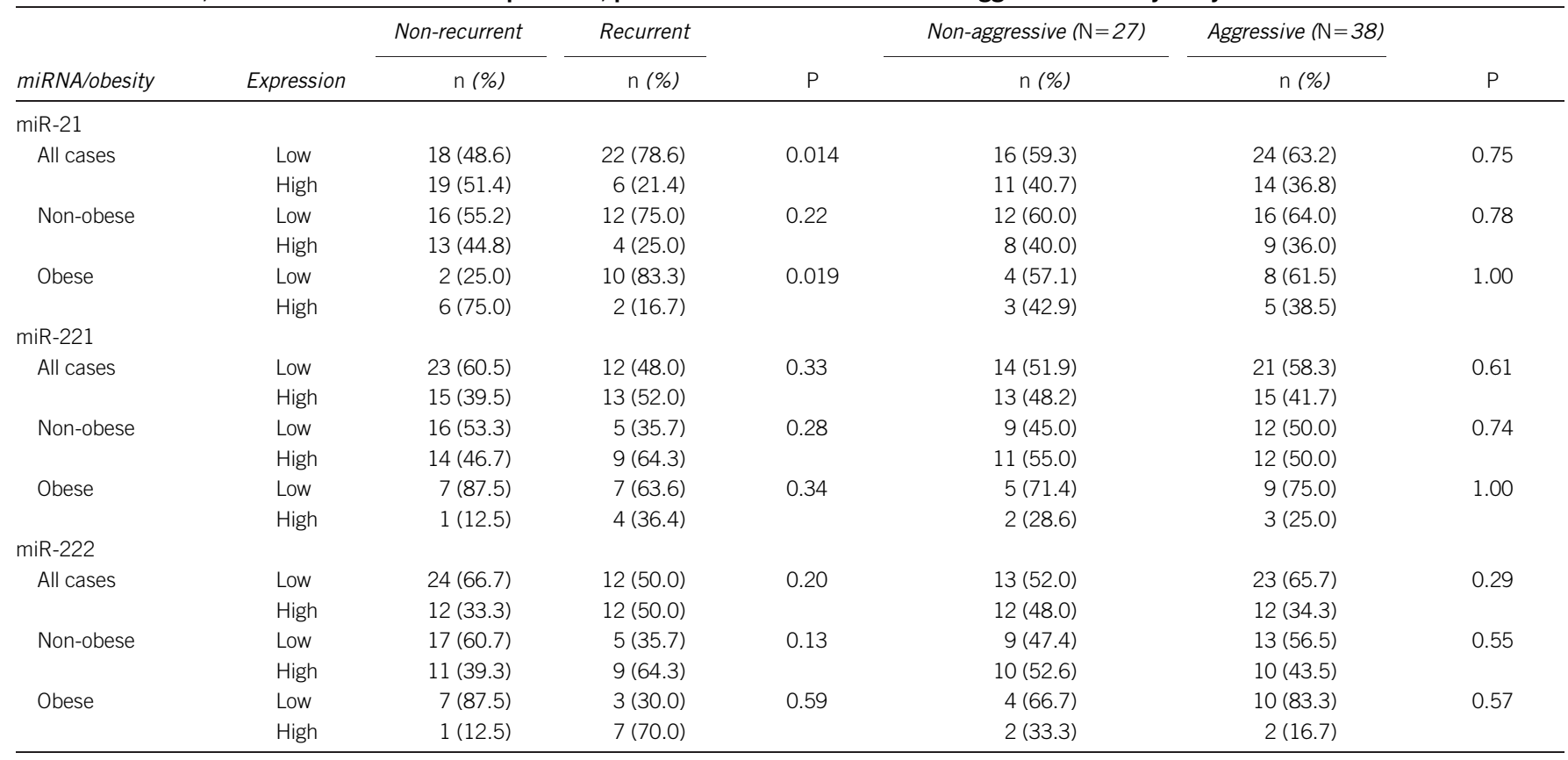

We did not observe a significant association between miRNA expression and aggressive prostate cancer (Tables 2 and $\mathbf{3}$ ).

\section{DISCUSSION}

In this study, we evaluated the associations between prostate cancer recurrence and the expression of miR-21, miR-221 and miR-222; and then examined whether the associations were modified by obesity. We observed an association between miR-21 expression and prostate cancer recurrence and report for the first time that the association between miR-21 expression and prostate cancer recurrence is more prominent in obese than non-obese cases.

Differential expression of miR-21 has been reported in several cancers, including prostate cancer, and has been associated with aggressive disease or outcome in prostate cancer. Li et al. ${ }^{16}$ recently showed that differential expression of miR-21 was associated with recurrencefree survival in prostate cancer patients treated with radical prostatectomy. Furthermore, miR-21 is differentially expressed in castration resistant prostate cancer compared with benign prostate hyperplasia. ${ }^{17}$ Recently, Zhang et al. ${ }^{18}$ observed higher levels of miR-21 in patients with androgen dependent prostate cancer compared to

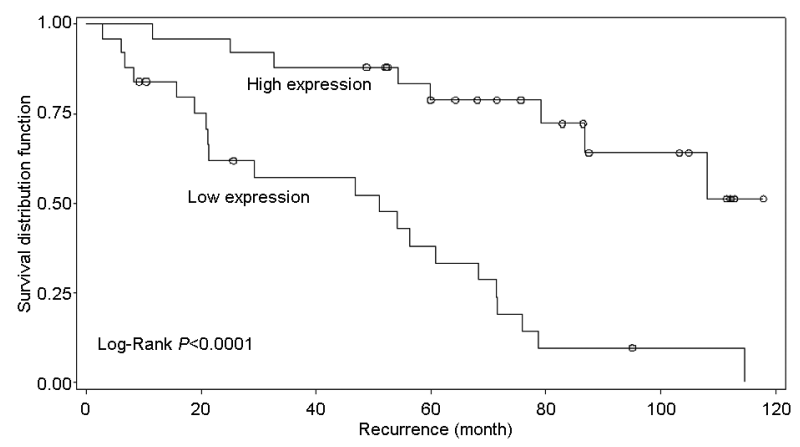

Figure 1 Kaplan-Meier plot for miR-21 expression and prostate cancer recurrence for all patients. hormone refractory patients (especially in those resistant to docetaxel-based chemotherapy) and suggested that miR-21 may be a marker for the transition to hormone refractory disease and a potential predictor for response to therapy in prostate cancer patients. Elevated plasma levels of miR-21 has been associated with aggressive prostate cancer ${ }^{19}$ and serum levels of miR-21 are elevated in hormone refractory prostate cancer patients compared to patients with benign prostatic hyperplasia. ${ }^{20}$ Additionally, miR-21 expression levels are elevated in prostate tumor tissue compared to adjacent normal tissue. $^{21,22}$ However, our study showed that low expression of miR-21 was associated with poor recurrence free survival and we did not confirm a positive association between miR-21 expression and aggressive prostate cancer. The discrepancy in our findings compared to the study by Li et al. ${ }^{16}$ that showed an association between a high expression of miR-21 and poor recurrence-free survival may potentially be attributed to the different proportions of obese cases in the studies. Due to the strong positive association between miRNA expression and prostate cancer recurrence observed in our study, it is plausible that a high proportion of obese cases in a study may show a positive association in the overall cases. Unfortunately, data on the proportion of obese cases in the study by Li et al. ${ }^{16}$ was not available.

Obesity is an important predictor for prostate cancer recurrence and a recent meta-analysis including 11 studies and over 4000 cases that developed recurrence after radical prostatectomy showed an increased risk $(\mathrm{OR}=1.25,95 \% \mathrm{CI}=1.12-1.40)$ of recurrence associated with higher BMI. ${ }^{12}$ Emerging evidence suggests miRNAs play a key role in the pathological development of obesity by affecting adipogenesis, which contribute to increased adipose tissue mass in obesity. ${ }^{23-26}$ Several miRNAs have been identified that affect adipogenesis by targeting adipogenesis regulators such as PPAR $\gamma$ and $\mathrm{C} / \mathrm{EBP} \alpha^{11,27,28}$ and perturbations in this regulation is linked to obesity. ${ }^{29}$ miR-21 is upregulated in subcutaneous adipose tissue in human obesity and positively correlated with BMI. ${ }^{30}$ Kim et al. ${ }^{31}$ showed that miR-21 controls the adipogenic differentiation of human adipose tissue-derived mesenchymal stem cells and the same group recently showed that the expression of miR-21 in white adipose tissues in an obesity-induced mouse 
Table 3 Association of miR-21, miR-221 and miR-222 expression with prostate cancer recurrence free survival and aggressiveness

\begin{tabular}{|c|c|c|c|c|c|c|}
\hline & \multicolumn{4}{|c|}{ Recurrence } & \multicolumn{2}{|c|}{ Aggressiveness } \\
\hline & Age adjusted $^{\mathrm{a}} \mathrm{HR}(95 \% \mathrm{Cl})$ & $P$ & Multivariate ${ }^{b}$ HR $(95 \%$ CI) & $\mathrm{P}$ & OR $(95 \% \mathrm{Cl})$ & $P$ \\
\hline \multicolumn{7}{|l|}{ miR-21 } \\
\hline All cases & $3.31(1.32-8.34)$ & 0.011 & $1.99(0.70-5.64)$ & 0.20 & $1.53(0.52-4.52)$ & 0.45 \\
\hline Non-obese cases & $2.26(0.69-7.34)$ & 0.18 & $1.28(0.30-5.49)$ & 0.74 & $1.80(0.46-7.00)$ & 0.40 \\
\hline Obese cases & $5.40(1.16-25.10)$ & 0.031 & $6.15(1.04-36.48)$ & 0.045 & $1.20(0.19-7.78)$ & 0.85 \\
\hline \multicolumn{7}{|l|}{ miR-221 } \\
\hline All cases & $0.71(0.32-1.61)$ & 0.42 & $0.56(0.21-1.50)$ & 0.25 & $1.28(0.47-3.50)$ & 0.64 \\
\hline Non-obese cases & $0.50(0.15-1.66)$ & 0.26 & $0.40(0.09-1.84)$ & 0.24 & $1.23(0.37-4.05)$ & 0.74 \\
\hline Obese cases & $0.77(0.23-2.66)$ & 0.68 & $0.46(0.10-2.22)$ & 0.33 & $1.26(0.15-10.52)$ & 0.83 \\
\hline \multicolumn{7}{|l|}{ miR-222 } \\
\hline All cases & $0.51(0.22-1.18)$ & 0.12 & $0.39(0.14-1.15)$ & 0.09 & $1.62(0.56-4.74)$ & 0.37 \\
\hline Non-obese cases & $0.37(0.11-1.20)$ & 0.10 & $0.37(0.09-1.59)$ & 0.18 & $1.36(0.39-4.68)$ & 0.63 \\
\hline Obese cases & $0.55(0.12-2.47)$ & 0.44 & $0.46(0.07-3.19)$ & 0.44 & $2.26(0.22-23.53)$ & 0.50 \\
\hline
\end{tabular}

${ }^{\text {a }}$ Adjusted for age at diagnosis (continuous).

${ }^{\mathrm{b}}$ Adjusted for age at diagnosis (continuous), Gleason score ( $<7$ vs. $\geqslant 7$ ) and stage ( 1 and 2 vs. 3 and 4 ).

model correlated with adipocyte number, suggesting the role of miR21 in adipogenesis. In mice fed with a high-fat diet, up-regulation of miR-21 and miR-222 was observed during the development of obesity. ${ }^{32}$ Recently, Terao et al. ${ }^{33}$ reported miR-21 target genes using gene expression microarrays in combination with a bioinformatic approach. Among 481 potential target genes identified, there were genes involved in the adipogenesis pathway, such as APOA1, IGFBP4, IGF2 and $A P O M$. Interestingly, some of these genes are also involved in prostate cancer progression. For example, in vivo studies have shown that overexpression of IGFBP4 decreases the growth of prostate cancer. ${ }^{34}$ The expression of IGF axis, including IGF2, is enhanced during normal prostate epithelial differentiation ${ }^{35}$ and aberrant expression of IGF2 has recently been suggested as an early event that contributes to prostate cancer progression. ${ }^{36}$

The mechanism linking obesity, miRNA expression and prostate cancer recurrence is not completely understood. However, based on the existing literature, it is plausible that expression of miR- 21 in obese prostate cancer patients may influence the expression of angiogenesis factors, such as HIF-1 $\alpha$ and VEGF, which are associated with prostate cancer progression. Recent in vitro studies show that overexpression of miR-21 in a prostate cancer cell line (DU145) increased expression of $H I F-1 \alpha$ and VEGF. ${ }^{37}$

This study has limitations. The study included a small sample size and the results need to be interpreted with caution. In addition, the study included only Caucasians and we could not determine if the observed associations differ by ethnicity. Future larger studies including populations of other ethnicities are therefore warranted to confirm these findings and to determine ethnic differences. Furthermore, data on the gene expression profiles of our study participants were not available to correlate miRNA to target mRNA. Studies including both miRNA and mRNA expression on prostate cancer recurrence are required to investigate this correlation.

In summary, our findings suggest that miR-21 is associated with prostate cancer recurrence after radical prostatectomy and show for the first time that the differential expression is more pronounced in obese than non-obese cases. Future studies are warranted to confirm these initial findings and to elucidate the mechanisms involved.

\section{AUTHOR CONTRIBUTIONS}

EKA and JYP conceived and designed the experiments. EKA, EA and HP performed the experiments. EKA analyzed the data. JPS, AH and JYP contributed reagents and tissues. EKA and JYP wrote the paper.

\section{COMPETING FINANCIAL INTERESTS}

All authors declare that there are no competing financial interests.

\section{ACKNOWLEDGMENTS}

This research was supported in part by the National Cancer Institute grant (R01CA128813) for JYP and a cancer prevention fellowship for EKA by the National Cancer Institute (R25T CA147832).

1 Siegel R, Naishadham D, Jemal A. Cancer statistics, 2012. CA Cancer J Clin 2012 62: 10-29.

2 Cancer facts and figures. Atlanta, GA: American Cancer Society, 2010.

3 Siddiqui SA, Inman BA, Sengupta S, Slezak JM, Bergstralh EJ et al. Obesity and survival after radical prostatectomy: a 10-year prospective cohort study. Cancer 2006; 107 521-9.

4 Amankwah EK, Park JY. miRNA and human prostate cancer. In: Sahu SC (ed). Toxicology and Epigenetics. 1st edn. Hoboken: Wiley, John and Sons, Incorporated 2012.

5 Fendler A, Jung M, Stephan C, Honey RJ, Stewart RJ et al. miRNAs can predict prostate cancer biochemical relapse and are involved in tumor progression. Int $J$ Oncol 2011; 39: 1183-92.

6 Leite KR, Tomiyama A, Reis ST, Sousa-Canavez JM, Sanudo A et al. MicroRNA-100 expression is independently related to biochemical recurrence of prostate cancer. J Urol 2011; 185: 1118-22.

7 Long $\mathrm{Q}$, Johnson BA, Osunkoya AO, Lai $\mathrm{YH}$, Zhou W et al. Protein-coding and microRNA biomarkers of recurrence of prostate cancer following radical prostatectomy. Am J Pathol 2011; 179: 46-54.

8 Schaefer A, Jung M, Mollenkopf HJ, Wagner I, Stephan C et al. Diagnostic and prognostic implications of microRNA profiling in prostate carcinoma. Int $\mathrm{J}$ Cancer 2010; 126: 1166-76.

9 Spahn M, Kneitz S, Scholz CJ, Stenger N, Rudiger T et al. Expression of microRNA221 is progressively reduced in aggressive prostate cancer and metastasis and predicts clinical recurrence. Int J Cancer 2010; 127: 394-403.

10 Tong AW, Fulgham P, Jay C, Chen P, Khalil I et al. MicroRNA profile analysis of human prostate cancers. Cancer Gene Ther 2009; 16: 206-16.

11 Alexander R, Lodish H, Sun L. MicroRNAs in adipogenesis and as therapeutic targets for obesity. Expert Opin Ther Targets 2011; 15: 623-36.

12 Cao Y, Ma J. Body mass index, prostate cancer-specific mortality, and biochemical recurrence: a systematic review and meta-analysis. Cancer Prev Res (Phila) 2011; 4: 486-501.

13 Hudson RS, Yi M, Esposito D, Glynn SA, Starks AM et al. MicroRNA-106b-25 cluster expression is associated with early disease recurrence and targets caspase- 7 and focal adhesion in human prostate cancer. Oncogene e-pub ahead of print 17 September 2012.

14 Hudson RS, Yi M, Esposito D, Watkins SK, Hurwitz AA et al. MicroRNA-1 is a candidate tumor suppressor and prognostic marker in human prostate cancer. Nucleic Acids Res 2012; 40: 3689-703.

15 Zhou X, Marian C, Makambi KH, Kosti O, Kallakury BV et al. MicroRNA-9 as potential biomarker for breast cancer local recurrence and tumor estrogen receptor status. PLOS One 2012; 7: e39011.

$16 \mathrm{Li} \mathrm{T,} \mathrm{Li} \mathrm{RS,} \mathrm{Li} \mathrm{YH,} \mathrm{Zhong} \mathrm{S,} \mathrm{Chen} \mathrm{YY} \mathrm{et} \mathrm{al.} \mathrm{miR-21} \mathrm{as} \mathrm{an} \mathrm{independent} \mathrm{biochemical}$ recurrence predictor and potential therapeutic target for prostate cancer. $J$ Urol 2012 187: 1466-72. 
neg

miRA and prostate cancer recurrence by obesity status

EK Amankwah et al

230

17 Jalava SE, Urbanucci A, Latonen L, Watering KK, Sahu B et al. Androgen-regulated miR-32 targets BTG2 and is overexpressed in castration-resistant prostate cancer. Oncogene 2012; 31: 4460-71.

18 Chang HL, Yang LF, Zhu Y, Yo XD, Zhang SL et al. Serum miRNA-21: elevated levels in patients with metastatic hormone-refractory prostate cancer and potential predictive factor for the efficacy of docetaxel-based chemotherapy. Prostate $2011 ; 71$ : 326-31.

19 Shin J, Hruby GW, McKiernan JM, Gurvich I, Lipsky MJ et al. Dysregulation of circulating microRNAs and prediction of aggressive prostate cancer. Prostate 2012; 72: 1469-77.

20 Chang HL, Yang LF, Zhu Y, Yo XD, Chang SL et al. Serum miRNA-21: elevated levels in patients with metastatic hormone-refractory prostate cancer and potential predictive factor for the efficacy of docetaxel-based chemotherapy. Prostate 2011; 71: 326-31.

21 Ribas J, Ni X, Hefner M, Wentzel EA, Salmasi AH et al. miR-21: an androgen receptorregulated microRNA that promotes hormone-dependent and hormone-independent prostate cancer growth. Cancer Res 2009; 69: 7165-9.

22 Ribas J, Lupold SE. The transcriptional regulation of miR-21, its multiple transcripts, and their implication in prostate cancer. Cell Cycle 2010; 9: 923-9.

23 Esau C, Davis S, Murray SF, Mu XX, Pandey SK et al. miR-122 regulation of lipid metabolism revealed by in vino antisense targeting. Cell Metal 2006; 3: 87-98.

24 Lin Q, Gao Z, Alarcon RM, Ye J, Pun Z. A role of miR-27 in the regulation of adipogenesis. FEBS J 2009; 276: 2348-58.

$25 \mathrm{Xie} \mathrm{H}$, Kim B, Lodish HF. MicroRNAs induced during adipogenesis that accelerate fat cell development are downregulated in obesity. Diabetes 2009; 58: 1050-7.

26 Xe H, Sun L, Lodish HF. Targeting microRNAs in obesity. Expert Spin Then Targets 2009; 13: 1227-38.

27 Hulsmans $M$, de Keyzer D, Holvoet P. MicroRNAs regulating oxidative stress and inflammation in relation to obesity and atherosclerosis. FASEB J 2011; 25: 2515-27.

28 Parr P, Serra F, Palou A. Expression of adipose microRNAs is sensitive to dietary conjugated linoleic acid treatment in mice. PLo One 2010; 5: e13005.
29 Lee EK, Lee MJ, Abdelmohsen K, Kim W, Kim MM et al. miR-130 suppresses adipogenesis by inhibiting peroxisome proliferator-activated receptor gamma expression. Mol Cell Biol 2011; 31: 626-38.

30 Keller P, Gburcik V, Petrovic N, Gallagher IJ, Nedergaard J et al. Gene-chip studies of adipogenesis-regulated microRNAs in mouse primary adipocytes and human obesity. BMC Endocr Discord 2011; 11: 7.

31 Kim YJ, Huang SH, Che HH, Shin KK, Be YC. MicroRNA 21 regulates the proliferation of human adipose tissue-derived mesenchymal stem cells and high-fat diet-induced obesity alters microRNA 21 expression in white adipose tissues. J Cell Physiol 2012; 227: 183-93.

32 Chartoumpekis DV, Zaravinos A, Zeros PG, Iskrenova RP, Psyrogiannis Al et al. Differential expression of microRNAs in adipose tissue after long-term high-fat dietinduced obesity in mice. PLo One 2012; 7: e34872.

33 Terao M, Fratelli M, Kurosaki M, Zanetti A, Guarnaccia V et al. Induction of miR-21 by retinoic acid in estrogen receptor-positive breast carcinoma cells: biological correlates and molecular targets. J Biol Chem 2011; 286: 4027-42.

34 Durai R, Davies M, Yang W, Yang SY, Seifalian A et al. Biology of insulin-like growth factor binding protein-4 and its role in cancer (review). Int J Oncol 2006; 28: 131725.

35 Massoner P, Ladurner Rennau M, Heidegger I, Kloss-Brandstätter A, Summered M et al. Expression of the IGF axis is decreased in local prostate cancer but enhanced after benign prostate epithelial differentiation and TGF-beta treatment. Am J Pathol 2011; 179: 2905-19.

36 Ribarska T, Bastion KM, Koch A, Schulz WA. Specific changes in the expression of imprinted genes in prostate cancer-implications for cancer progression and epigenetic regulation. Asian J Androl 2012; 14: 436-50.

37 Li LZ, Li C, Chen Q, Jung Y, Carpenter R et al. MiR-21 induced angiogenesis through AKT and ERK activation and HIF-1alpha expression. PLo One 2011; 6: e19139.

Asian Journal of Andrology 